\title{
Development and Validation of a Gas Chromatographic Method for Identification and Quantification of Terpene Trilactones in Ginkgo biloba L. Extract and Pharmaceutical Preparations
}

\author{
Lotfi Sayadi ${ }^{*}, 1,2$, Ines Missaoui ${ }^{3}$, Bassem Jamoussi ${ }^{*}, 4$ and Abdelmanef Abderraba ${ }^{4}$ \\ ${ }^{I}$ Faculté des Sciences de Tunis, Département de Chimie, 1060 Tunis, Tunisia \\ ${ }^{2}$ Opalia Pharma, Kalaat Andalous, 2022 Ariana, Tunisia \\ ${ }^{3}$ Institut Supérieur de l'Education et de la Formation Continue, Département des Sciences Physiques, 43 rue de la \\ Liberté, 2019 le Bardo, Tunisia \\ ${ }^{4}$ Laboratoire Physicochimie des Matériaux, IPEST, BP51, La Marsa 2070, Tunisia
}

\begin{abstract}
A gas chromatographic method was developed and validated for the determination of bilobalide and ginkgolides A, B, C and J in Ginkgo biloba L. The Ginkgo terpene trilactones have been dissolved in 57\% of pure ethanol in purified water and separated by extraction with a mixture of toluene/ethyl acetate. After evaporation of the solvent with nitrogen and derivatization, these chemical markers have been quantified using GC-FID. The intra-day RSD was ranged from $1.13 \%$ for bilobalide to $2.34 \%$ in case of ginkgolides. The inter-day RSD was ranged from $2.54 \%$ and $3.68 \%$ for, bilobalide and ginkgolides respectively. Mean recoveries were $99.78 \% \pm 0.82 \%$ and $99.48 \% \pm 0.86 \%$ for ginkgolides and bilobalide, respectively. Based on chromatograms, the response of the terpene trilactones was linearly dependent on the terpene concentration, i.e. linear calibration curve was found in the range of the method and was applied to analyze standard extract (containing 5-7\% total terpenoids and $22-25 \%$ total flavonoids) as working standard and for the analysis of six Ginkgo pharmaceutical products offered on the market.
\end{abstract}

Keywords: Ginkgo biloba, bilobalide, ginkgolides, gas chromatography.

\section{INTRODUCTION}

The extracts of Ginkgo biloba L. (GBE) are the most frequently sold phytopharmacological products in America and Europe and largely used as a supplement of lunching in Japan, China as well in the United States [1-3]. The flavonoide glycosides and terpene trilactones presented in Ginkgo are considered as interesting molecules from a pharmaceutical point of view. The ginkgolides A, B, C and J and the bilobalide are the names of terpenes in GBE. The first ones were regarded as diterpenes and the bilobalide as sesquiterpene (C15 closed terpene). Structures of ginkgolides $\mathrm{A}, \mathrm{B}$, and $\mathrm{C}$ were determined by two Japanese groups in the sixties of past century [4-6] whereas the structures of the bilobalide [7] and ginkgolide J [8] were discovered few years later. They are highly oxidable compounds, especially in the presence of air's oxygen.

The interest of the analysis of ginkgolides had increased in the eighties because of the need to explain their exact therapeutic action and the increase in the cost of the extracts of Ginkgo.

Some clinical studies showed the effect of Ginkgo in the improvement of peripheral and cerebrovascular circulation

*Address correspondence to these authors at the (LS) Faculté des Sciences de Tunis, Département de Chimie, 1060 Tunis, Tunisia; Tel : 0021697733114 ; Fax : (216) 71797677; E-mail: inglotfi@gmail.com; (BJ) Laboratoire Physicochimie des Matériaux, IPEST, BP51, La Marsa 2070, Tunisia; Tel: 0021698340614 ; E-mail: jamoussiVbassem@yahoo.fr
$[9,10]$ and treatment of the neurosensory problems [11]. In addition, it was noticed, that a significant improvement of the skin blood perfusion and blood's viscosity and elasticity fall [12], and consequently, the increase in the transport of oxygen and glucose towards the various bodies and with the brain particularly $[13,14]$.

The extracts of Ginkgos' sheets were largely used for the treatment of the cerebral insufficiency by modulation of the cerebral energy metabolism and inhibition of the acetylcholinesterase, thanks to terpenes trilactones $[15,16]$. The GBE were also used to fight with variety of neurological energy disorders of the Alzheimer disease passing through the depression, lack of attention and temporary loss of the memory [17-19]. GBE is categorized, like vitamins C, B and beta-carotene, as the diet supplementary agents with antioxidant and free anti-radicals properties [20,21]. The extracts of the Ginkgos' sheets are also largely applied to treat the cerebral vascular ischemia [22]. Indeed, these extracts can correct the cerebral damage caused by an ischemia by improving blood circulation and increasing the cerebral blood flow rich in glucose and oxygen [14]. The ginkgolides' structures, including bilobalide are shown in Fig. (1).

Various analytical techniques were used to quantify or identify the terpene trilactones in the standardized extracts and the products of Ginkgo. The ginkgolides are non-volatile compounds and polar in nature. Therefore, they were suitably analyzed by reverse phase high performance liquid 


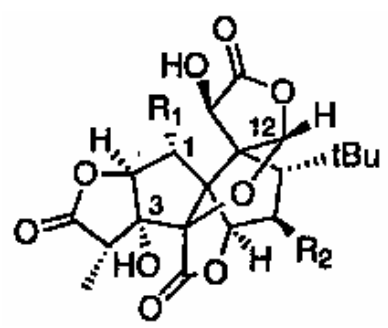

Ginkgolides

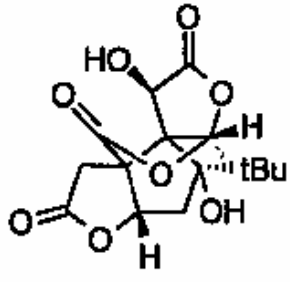

Bilobalide

Fig. (1). Chemical structures of the terpenes trilactones in Ginkgo biloba. $\mathrm{GA}: \mathrm{R}_{1}=\mathrm{R}_{2}=\mathrm{H} ; \mathrm{GB}: \mathrm{R}_{1}=\mathrm{OH}, \mathrm{R}_{2}=\mathrm{H} ; \mathrm{GC}: \mathrm{R}_{1}=\mathrm{R}_{2}=\mathrm{OH}$; GJ : $\mathrm{R}_{1}=\mathrm{H}, \mathrm{R}_{2}=\mathrm{OH}$.

chromatography (RP-HPLC). However, they have poor chromophores and can only be monitored at low and non selective wavelengths (190-220 nm). This makes UV detection difficult, interfered with trace impurities especially when analyzing crude Ginkgo extracts and extensive cleanup procedures that are usually required as prior to analyze leaf extracts and dosage forms [23-26]. In spite of this detection difficulty, an Egyptian group validated and published a RP-HPLC method using UV detection at $220 \mathrm{~nm}$ [27]. A better approach for the HPLC detection was refractive index (RI) detection but unstable baseline problems and low sensitivity detract from its selectivity $[28,29]$. Mass spectrometry (MS) coupled to LC or GC (LCMS, GC-MS) was used and has proved a good detection due to its sensitivity and low limits of detection and quantification [3,23,30-34]. The terpene trilactones have been analyzed by HPLC by using evaporative light scattering detection (ELSD) $[25,35,36]$ or by gas chromatography by using flame ionization detection (GC-FID) [29,30]. Reverseflow micellar electrokinetic chromatography (RF-MEKC) was applied by Dubber and Kanfer [37] and NMR by Van Beek et al. [38]. A new method, using LC-atmospheric pressure chemical ionization (APCI)-MS in the negative ionization has been used to analyse ginkgolides and bilobalide in food products $[39,40]$. However, the methods using instruments like mass spectrometry MS, NMR, APCIMS and RF-MEKC are very expensive and increase analysis cost and might be not available in routine QC laboratories. HPLC-ELSD due to the simplification of sample preparation and its inexpensive detection and GC-FID due to its availability and its inexpensive cost were the economic choices for routine QC laboratories. However, the ELSD process needs nitrogen to make nebulization and an oven to evaporate the solvent or the mobile phase. In the present paper, a quantitative GC-FID method is described for the determination of terpene trilactones (ginkgolides A, B, C, J and bilobalide) in Ginkgo biloba extracts and products by using a simple purification and sample derivatization before the analysis and a fast run, only 13 minutes for one injection as compared to those in literature [34].

\section{MATERIALS AND METHODS}

\subsection{Reagents}

The used reagents were of analytical grade without previous purification. Standard reference compounds of ginkgolide A, ginkgolide B, bilobalide (terpene trilactones) and standardized Ginkgo biloba L. extract (raw material) were purchased from Indena (Italy), squalane and $\mathrm{N}, \mathrm{O}$ bis(trimethylsilyl)trifluoroacetamide (BSTFA) containing $1 \%$ trimethylchlorosilane (TMSC) and N,N-dimethylformamide (DMF) were purchased from Sigma (Germany), hexane was purchased from Carlo Erba (Italy), pure ethanol was purchased from Riedel-de-Haën (Germany) and toluene were purchased from Scharlau (Spain). Water was purified by osmosis system. Ginkgo products were provided by OPALIA laboratories and purchased from a local pharmacy in Hammam-Lif area (Tunisia).

\subsection{Standard Stock and Calibration Solutions}

Ginkgo biloba stock solution was prepared from standardized Ginkgo biloba L. extract by dissolution $57 \%$ of pure ethanol in purified water and was stored in refrigerator $\left(-4^{\circ} \mathrm{C}\right)$ for stability. The solution contained $40 \mathrm{mg} / \mathrm{mL}$ of Ginkgo biloba L. extract. Appropriate aliquots were taken from the stock solution and used in the extraction step. The quantities of Ginkgo biloba in the aliquots were used for linearity determination.

\subsection{Sample Preparation and Derivatization}

A $500 \mathrm{mg}$ of standardized Ginkgo biloba L. extract was taken for the analysis (standard stock solution). Samples of Ginkgo biloba extract products were prepared by taking $2 \mathrm{~mL}$ of the liquid forms in a dry and clean tube tests. Capsules' samples could be prepared by combining the contents of 15 capsules and taking the equivalent of $500 \mathrm{mg}$ of GBE from the mixture for analysis. Tablets' samples were prepared by crushing in a mortar 15 tablets and taking the equivalent of $500 \mathrm{mg}$ of GBE. Samples were dissolved in $12.5 \mathrm{~mL}$ of $57 \%$ of pure ethanol in purified water, sonicated for 20 minutes and vortexed every 5 minutes. A $2 \mathrm{~mL}$ of the clear solution were transferred into a dry tube tests in which one adds $500 \mu \mathrm{L}$ of squalane ( $1 \mathrm{mg} / \mathrm{mL}$ in hexane) as internal standard. The mixture was extracted with $4 \mathrm{~mL}$ of a mixture of toluene and ethyl acetate $(7 / 3)$ by vortexing for 1 minute. Then, $2 \mathrm{~mL}$ of the organic phase was transferred into a $10 \mathrm{~mL}$ head space vial and the solvent was evaporated by a nitrogen flow (a temperature between 40 and $50^{\circ} \mathrm{C}$ can be used for evaporating). To the residue, one was added $400 \mu \mathrm{L}$ of dimethylformamide and $400 \mu \mathrm{L}$ of mixture BSTFA/TMSC (99/1) which had been heated on the head space's four or another heat block at $100^{\circ} \mathrm{C}$ for 1 hour. After cooling, a $2 \mu \mathrm{L}$ of the derivatized sample was injected for GC-FID identification and quantification. The preparation time was estimated to one hour and the derivatized time was estimated to one hour and 25 minutes. 


\subsection{GC-FID Conditions}

The derivatized samples and derivatized calibration solutions were injected into a GC-FID system that consisted of an HP 5890 gas chromatograph with flame ionization detection (FID). Separations were accomplished with a fused-silica capillary column $(10 \mathrm{~m} \times 0.53 \mathrm{~mm})$ coated with $0.88 \mu \mathrm{m}$ layer of cross-linked methyl siloxane (HP-1). The injection and detection temperatures were $280^{\circ} \mathrm{C}$ and $300^{\circ} \mathrm{C}$ respectively. The injection mode is split with ratio of $2: 1$. The flow-rate of the carrier gas (nitrogen) was maintained at $3 \mathrm{~mL} / \mathrm{min}$. The oven temperature was initialled at $250^{\circ} \mathrm{C}$ for 2 minutes, and then increased to $300^{\circ} \mathrm{C}$ at $5^{\circ} \mathrm{C} / \mathrm{min}$, had been holding for 1 minute. The total analysis time was 13 minutes. The quantification was performed with flame ionisation detection with flows 400,45 and $25 \mathrm{~mL} / \mathrm{min}$ successively for compressed air, hydrogen and nitrogen as make-up.

\subsection{Calculations}

The recoveries' percentages $(R \%)$ of bilobalide, ginkgolides or sum of terpenes content were determined by the formula (1):

$R \%=\frac{A E}{A S} \times \frac{A I S}{A I E} \times \frac{M E}{M S} \times 100$

where AE (AS) is the peak area of bilobalide, sum of the ginkgolides or sum of terpenes in the sample (standard) chromatogram. AIE (AIS) is the peak area of internal standard (squalane) in the sample (standard) chromatogram. ME (MS) is the mass in mg of Ginkgo biloba in the sample (standard) preparation. The bilobalide, ginkgolides or sum of terpenes content in the analysed extract were determined by the formula (2):

$m g$ (terpene $) / 100 \mathrm{ml}=\frac{\%(\text { terpene })}{100} \times \frac{4}{100} \times T a \times 1000$

where $\%$ (terpene) is the percentage of bilobalide, ginkgolides or sum of terpenes and $\mathrm{Ta}$ is the assay of bilobalide, ginkgolides or sum of terpenes in the raw material (GBE) in \%.

\subsection{Method Validation}

\subsubsection{Standard and Sample Solutions Stability}

The terpenoid content of the standard and sample solutions were compared to freshly prepared reference solutions, prepared and stored in the refrigerator for 24 hours. The terpenoid content has not been changed during $24 \mathrm{~h}$.

\subsubsection{Calibration Curves}

Aliquots of the standard stock solution were taken and transferred to the test tubes. The contents of Ginkgo biloba L. extracts were $64,72,80,88$ and $96 \mathrm{mg}$ by tube corresponding to $60,80,100,120$ and $140 \%$ of theoretical quantity of Ginkgo biloba. Calibration curves were based on the sum of responses of ginkgolides, the responses of bilobalide and the sum of responses of all terpene trilactones. The calibration lines were constructed by plotting the areas of ginkgolides and bilobalide against their corresponding concentration.

\subsubsection{Precision and Accuracy}

The results of precision and accuracy determination were obtained from the recoveries of the ratios of found quantities (Ginkgo biloba in the presence of liquid forms matrix) to the introduced quantities (Ginkgo biloba only). The precision of the proposed method was verified by calculation of the repeatability's RSD and intermediate precision's RSD of six preparations done successively during one day and the following 3 consecutive days. The accuracy was determined by calculation of the mean recoveries $\pm \mathrm{SD}$ of five levels of concentrations.

\section{RESULTS AND DISCUSSION}

\subsection{Solubility and Extraction of Terpene Trilactones}

The $57 \%$ of pure ethanol in purified water was used to improve the solubility of terpene trilactones. This solvent, which is used in producing liquid pharmaceutical forms of Ginkgo, showed good solubility of terpenes. In the same case, pure methanol and $50 \%$ methanol in purified water were used to verify the solubility of terpenes and they gave the same results but methanol was toxic to the environment and health. The solvent used in this method was the best choice to dissolve Ginkgo biloba extracts as compared to other solvents in the literature like $1 \mathrm{~N} \mathrm{HCl}$ in $20 \%$ methanol [34] or $10 \% \quad \mathrm{NaH}_{2} \mathrm{PO}_{4}(\mathrm{~m} / \mathrm{v})$ with controling $\mathrm{pH}$ and temperature [30]. A mixture of toluene and ethyl acetate (7/3) was used to enhance the extraction of terpenes from Ginkgo biloba L. extract. It gave high recoveries (>98\%) and constant retention times as compared to other solvent extraction reported in the literature [30,34]. The advantage of this mixed solvent was that it would give good separation without centrifugation of the two solutions' phases if we analyzed liquid forms. There would be a problem of phases' separation if we used ethyl acetate only [41] or in the mixture with tetrahydrofurane [30] to the extraction of terpenes from Ginkgo biloba in liquid forms.

\subsection{Derivatization Reaction of Terpenes}

The derivatization reaction of terpenes trilactones was based on a direct addition of the reagent BSTFA/TMSC $(99 / 1)$ in the presence of DMF to the dried residue obtained with a nitrogen's flow at a temperature of $100^{\circ} \mathrm{C}$ in 1 hour in a block four (a four of head space apparatus can be used). This temperature was used less than other temperatures as $120^{\circ} \mathrm{C}$ [30] or $115^{\circ} \mathrm{C} \mathrm{[34]} \mathrm{used} \mathrm{before} \mathrm{to} \mathrm{avoid} \mathrm{degradation}$ of some compounds. The time of the derivatization reaction was selected to be 1 hour which was optimized and was less than many other periods [41]. The use of DMF in the reaction was better than the use of pyridine. Indeed, currently the pyridine is evaluated as a possible carcinogenic agent and causes troubles for sexual activity $[41,42]$. The mixture of BSTFA/TMSC (99/1) was the derivatization reagent more frequently used by researchers. Then, it was selected in this proposed method. These conditions gave good repeatability of responses and retention times of analytes.

\subsection{Identification of Terpene Trilactones}

Fig. (2) shows a typical separation of bilobalide, ginkgolides A, J, B, C and the internal standard squalane in 13 minutes only. As compared to other chromatograms 


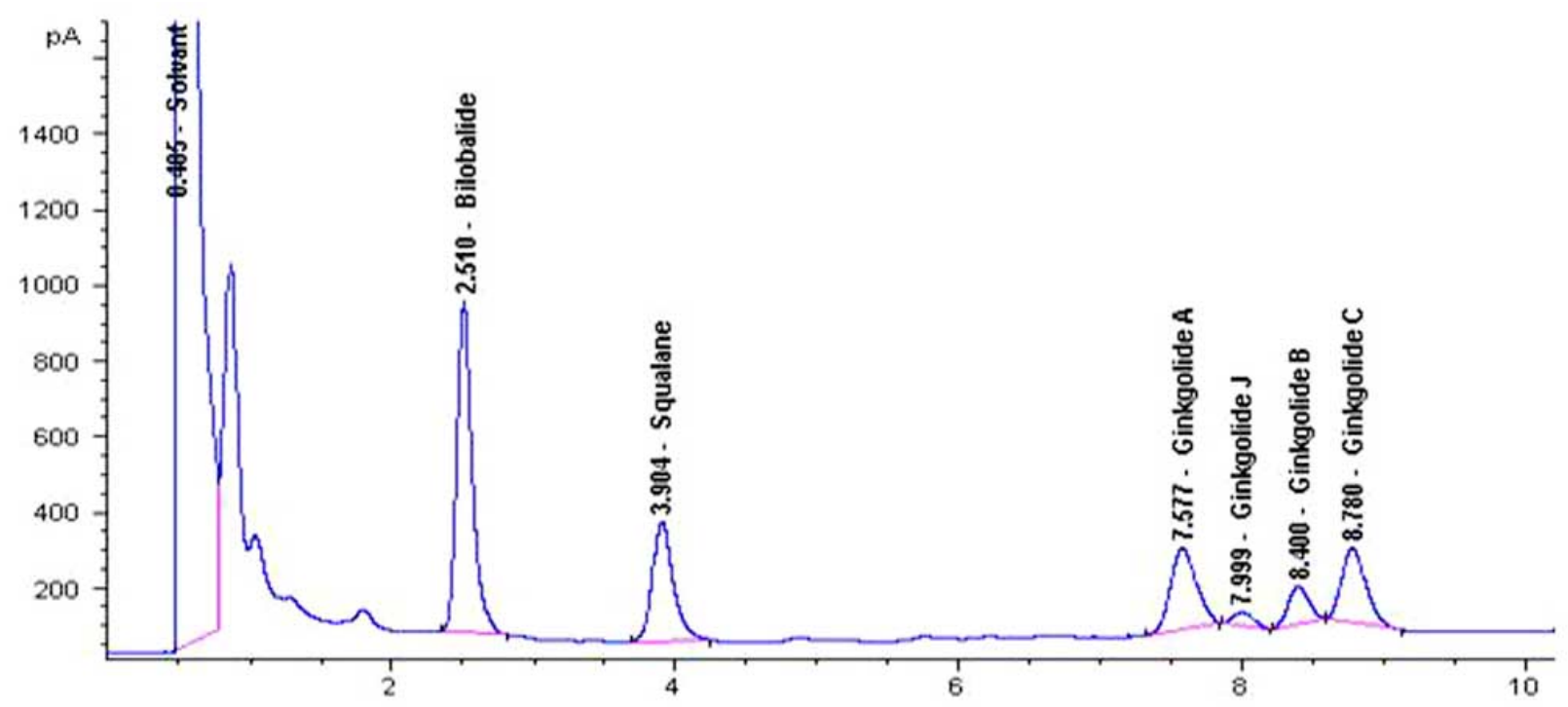

Fig. (2). A typical GC-FID chromatogram of a Ginkgo biloba L. extract.

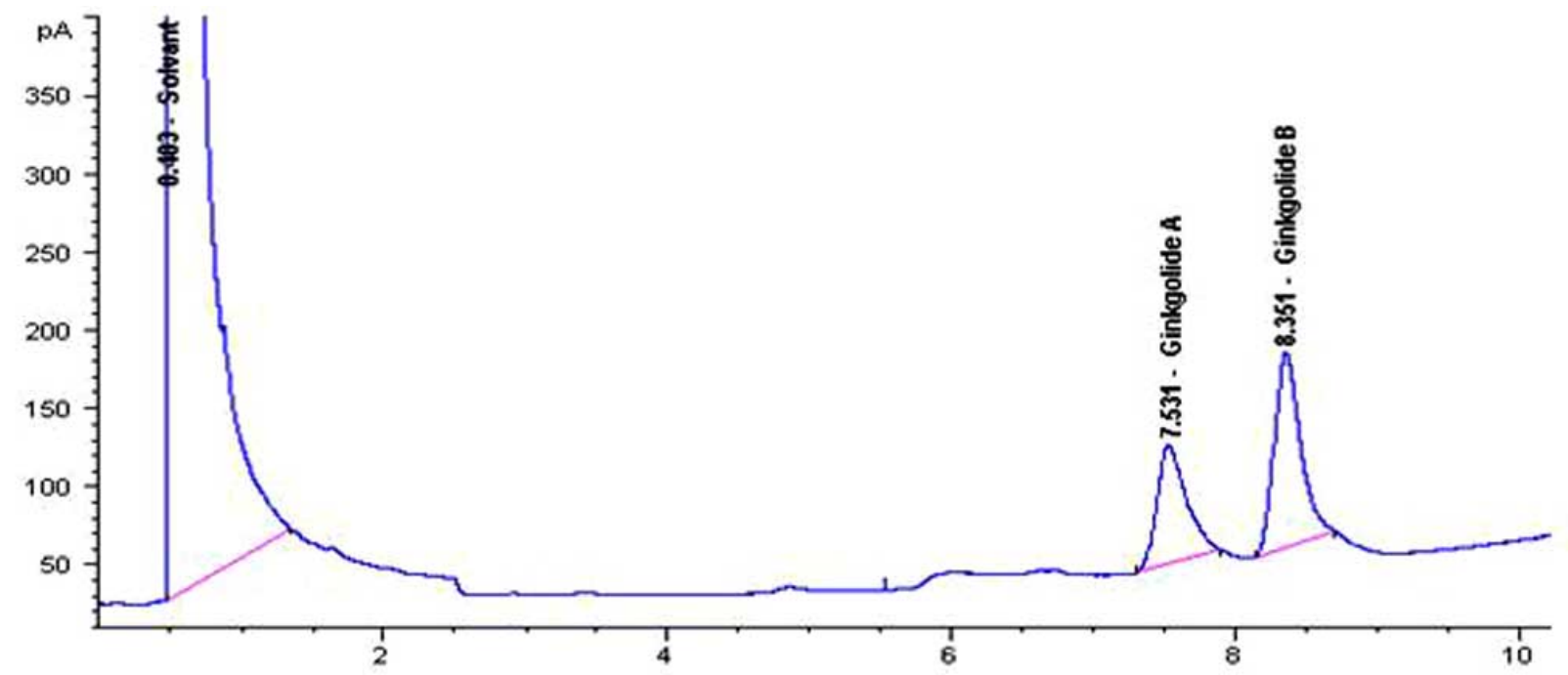

Fig. (3). A GC-FID chromatogram of ginkgolides A and B.

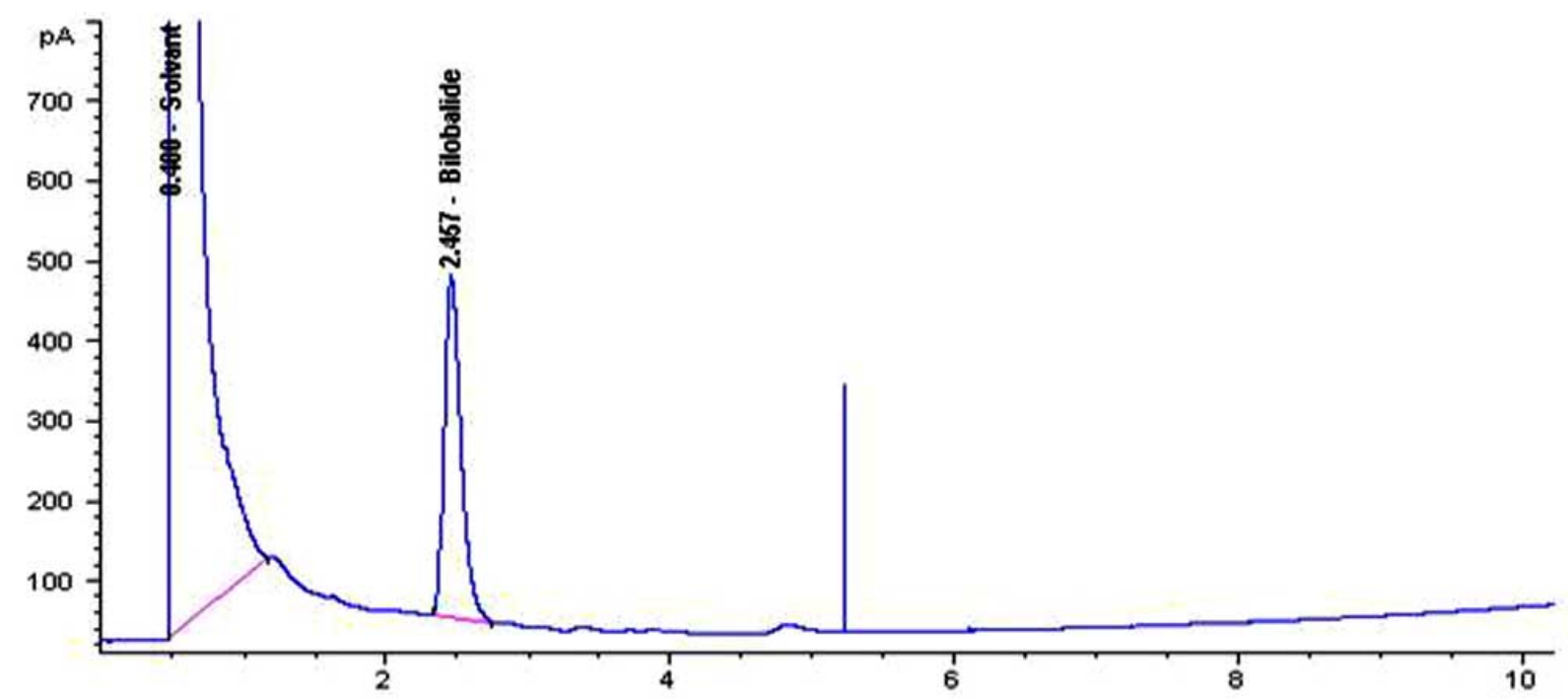

Fig. (4). A GC-FID chromatogram of bilobalide. 


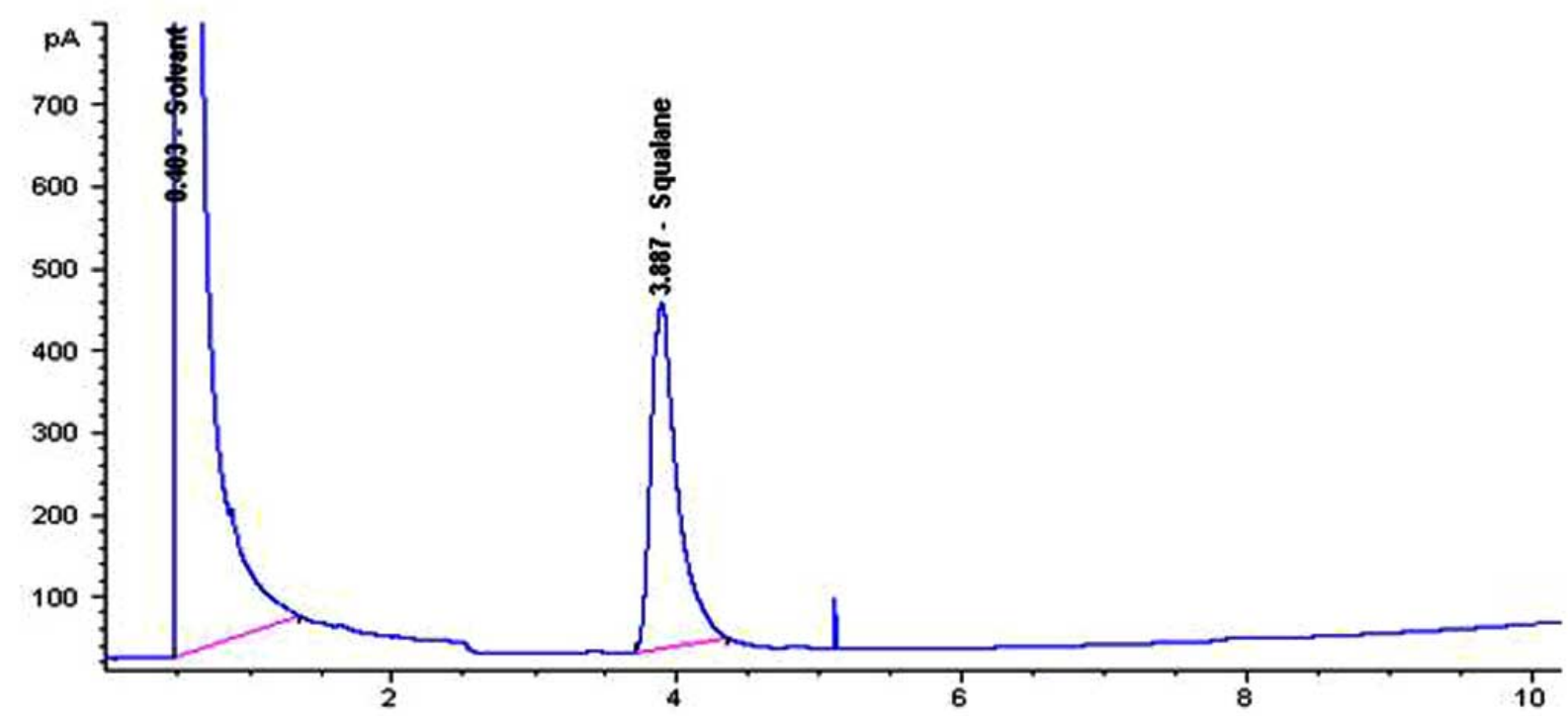

Fig. (5). A GC-FID chromatogram of squalane.

obtained before, the separations were longer (55 or 30 minutes) $[42,43]$. The terpene trilactones were identified by comparing in first step the retention times of the sample peaks to reference peaks (ginkgolide A, ginkgolide B and bilobalide) (Figs. 3-5) and in second step to the peaks in chromatogram obtained by Lang et al. [30] (ginkgolide $\mathrm{J}$ and ginkgolide $\mathrm{C}$ which were not available in our laboratory). The responses were better and higher than those obtained before by GC-FID [30] and the resolutions between peaks were above 1 . The retention times of peaks were stable and the standard deviation coefficient of six successive injections was less than $0.5 \%$.

\subsection{Method Validation}

\subsubsection{Linearity and Interferences}

The linearity of the method was verified by calculation of the coefficient of regression of the calibration curves. Therefore, $\mathrm{R}^{2}$ were above 0.9979 for all calibration curves. The calculation of t-Student value of comparison of calibration curves of Ginkgo biloba only and in the presence of the matrix of the liquid form shows that there are no effects of matrix on the response of terpenes. The formulas (3) and (4) were used in this test:

$$
\begin{aligned}
& t_{s}=\left|\frac{a_{11}-a_{12}}{\sqrt{S_{a 11}^{2}-S_{a 12}^{2}}}\right|<\mathrm{t}(0.05 ; 26) \\
& t_{0}=\left|\frac{a_{01}-a_{02}}{\sqrt{S_{a 01}^{2}+S_{a 02}^{2}}}\right|<\mathrm{t}(0.05 ; 26)
\end{aligned}
$$

where $t_{s}$ and $t_{0}$ are successively t-Student slope and t-Student zero value. $\mathrm{a}_{1 \mathrm{i}}$ and $\mathrm{Sa}_{1 \mathrm{i}}$ are the slopes and standard deviation of slopes, $\mathrm{a}_{0 \mathrm{i}}$ and $\mathrm{Sa}_{0 \mathrm{i}}$ are zero value and standard deviation of zero value. The results are presented in the Table 1.

\subsubsection{Precision and Accuracy}

The intra-day RSD's were $1.13 \%$ for bilobalide, $2.34 \%$ for ginkgolides and $1.54 \%$ for sum of terpenes. The inter-day RSD's were $2.54 \%$ for bilobalide, $3.68 \%$ for ginkgolides and $3.00 \%$ for sum of terpenes. The criterion of accuracy was verified by calculation of recoveries and accuracy's interval of ratios of found quantities (Ginkgo biloba in the presence of liquid forms matrix) on the introduced quantities (Ginkgo biloba only). Mean recoveries were $99.78 \pm 0.82 \%$ for ginkgolides and $99.48 \pm 0.86 \%$ for bilobalide. The recoveries obtained with the proposed method were better than obtained by other method reported before $[30,42,43]$.

\begin{tabular}{|c|c|c|c|c|c|}
\hline & & Regression Equation & $\mathbf{R}^{2}$ & ts calc. & to calc. \\
\hline \multirow{2}{*}{ Bilobalide } & GLE only & $y=69.70 x-161.2$ & 0.9986 & \multirow{2}{*}{1.172} & \multirow{2}{*}{1.781} \\
\hline & $\mathrm{GLE}+\mathrm{M}$ & $y=68.29 x-58.2$ & 0.9979 & & \\
\hline \multirow{2}{*}{ Ginkolides } & GLE only & $y=63.67 x-10.2$ & 0.9984 & \multirow{2}{*}{1.631} & \multirow{2}{*}{1.769} \\
\hline & $\mathrm{GLE}+\mathrm{M}$ & $y=65.78 x-230.2$ & 0.9980 & & \\
\hline \multirow{2}{*}{ Terpenes } & GLE only & $\mathrm{y}=133.37 \mathrm{x}-171.4$ & 0.9989 & \multirow{2}{*}{0.593} & \multirow{2}{*}{1.335} \\
\hline & $\mathrm{GLE}+\mathrm{M}$ & $y=134.07 x-288.4$ & 0.9999 & & \\
\hline
\end{tabular}

Table 1. Linearity Results of Calibration Curves of Terpenes

$\mathrm{t}(0.05 ; 26)=2.056$; M: matrix; GLE: Ginkgo biloba Leaf Extract. 
Table 2. Results of Terpene Recoveries in Pharmaceutical Products

\begin{tabular}{|c|c|c|c|c|c|c|}
\hline & Product 1* & Product 2* & Product 3* & Product 4* & Product 5* & Product 6* \\
\hline \hline Ginkgolides & $97.73 \pm 1.13 \%$ & $98.55 \pm 1.25 \%$ & $100.54 \pm 2.18 \%$ & $103.03 \pm 2.55 \%$ & $101.49 \pm 1.58 \%$ & $99.24 \pm 2.41 \%$ \\
\hline Bilobalide & $98.35 \pm 1.27 \%$ & $99.12 \pm 1.54 \%$ & $99.62 \pm 2.46 \%$ & $102.38 \pm 2.64 \%$ & $102.05 \pm 1.93 \%$ & $99.44 \pm 2.13 \%$ \\
\hline Sum of terpenes & $98.03 \pm 1.08 \%$ & $99.63 \pm 1.15 \%$ & $100.18 \pm 2.23 \%$ & $102.75 \pm 2.48 \%$ & $101.88 \pm 1.43 \%$ & $99.57 \pm 2.10 \%$ \\
\hline
\end{tabular}

\subsubsection{Limits of Detection (LOD) and Quantification (LOQ)}

The LOD was determined by the formula $3 \mathrm{Sb} /$ slope which $\mathrm{Sb}$ is the standard deviation of six measurements of the blank, whereas LOQ was determined by the formula $3 \mathrm{Sb} / \mathrm{slope}$. LOD and LOQ were successively $0.4 \mu \mathrm{g} / \mathrm{mL}$ and $1.3 \mu \mathrm{g} / \mathrm{mL}$.

\subsection{Analysis of Samples}

The proposed and validated method was applied to the analysis of six pharmaceutical samples containing Ginkgo biloba. In Table $\mathbf{2}$ the assay results were presented characterizing recoveries and content of bilobalide, ginkgolides and sum of terpenes.

These results showed, that obtained recoveries were between 97 and $103 \%$, and complied with specifications which were between 90 and $110 \%$, and the contents were all complied with specifications which were between 101 and $150 \mathrm{mg} / 100 \mathrm{~mL}$ for ginkgolides, in range of 94 and $141 \mathrm{mg} / \mathrm{mL}$ in case of bilobalide, and in range from 180 to $308 \mathrm{mg} / \mathrm{mL}$ for sum of terpenes.

\section{CONCLUSIONS}

Based on the above results, we consider that the developed and validated method could be applied for the determination of terpenes in Ginkgo biloba L. extract and in its pharmaceutical formulations with adequate linearity, reproducibility and recovery. Previously published methods for the determination of such terpenes needed to use an expensive laboratory equipments and materials, and also included a time-consuming extraction and derivatization steps. The proposed here method is an effective and simple and enables rapid determination of terpenes in GBE. This procedure presents a good linearity, accuracy, precision and no interference between excipients and other terpenes, as well as indicate sufficiently low LOD and LOQ. This method provides significant cost reduction and labor time advantages for routine quality control procedures performed daily in pharmaceutical laboratories and would be applied for more specific and high-throughput determination of terpenes in oral pharmaceutical forms (capsules or tablets) of Ginkgo biloba L. extract by further optimizing a little the preparation of the solutions by using an ultrasonic bath.

\section{ACKNOWLEDGEMENTS}

We would like to thank Dr. Alya El-Hedda, Opalia Pharma Director, Tunisia, for his constructive comments and discussion of this work.

\section{ABBREVIATIONS}

APCI-MS $=$ Atmospheric pressure chemical ionizationmass spectrometry
BSTFA $=\mathrm{N}, \mathrm{O}$-bis $($ trimethylsilyl)trifluoroacetamide

$\mathrm{DMF} \quad=\mathrm{N}, \mathrm{N}$-dimethylformamide

GBE $\quad=$ Gingko biloba extract

GC-FID $=$ Gas chromatograpy-flame ionization detector

LC-MS = Liquid chromatography - mass spectrometry

LOD $=$ Limit of detection

LOQ $=$ Limit of quantification

NMR $=$ Nuclear magnetic resonance

RF-MEKC $=$ Reverse flow micellar electrokinetic chromatography

RP-HPLC $=$ Reversed-phase high performance liquid chromatography

RSD $=$ Relative standard deviation

QC $=$ Quality control

TMSC $=$ Trimethylchlorosilane

\section{REFERENCES}

[1] United States Pharmacopoeia (USP29-NF24), Dietary supplements 3147, Rockville, MD, 2005, 2094.

[2] McKenna, D. J.; Jones, K.; Hughes, K. Efficacy, safety, and use of ginkgo biloba in clinical and preclinical applications. Altern. Ther., 2001, 7, 70 .

[3] Sticher, O. Quality of Ginkgo preparations. Planta Med., 1993, 59, 2 .

[4] Nakanishi, K.; Ginkgo Biloba, Van Beek, T.A., Ed.; Medicinal and Aromatic Plants-Industrial Profiles. Harwood: Amsterdam, 2000, vol. $12,517$.

[5] Nakanishi, K. The ginkgolides. Pure Appl. Chem., 1967, 14, 89

[6] Okabe, K.; Yamada, K.; Yamamura, S.; Takda, S. Ginkgolides. $J$. Chem. Soc. 1967, p. 2201.

[7] Nakanishi, K.; Habaguchi, H.; Nakadaira, Y.; Woods, M.C.; Maruyama, M.; Major, R.T.; Alauddin, M.; Patel, A.R.; Weinges, K.; Bahr, W. Structure of bilobalide, a rare tert-butyl containing sesquiterpenoid related to the C20-ginkgolides. J. Am. Chem. Soc., 1971, 93, 3544.

[8] Weinges, K.; Hepp, M.; Jaggy, H. Chemie der Ginkgolide, II. Isolierung und Strukturaufklärung eines neuen Ginkgolids. Liebigs Ann. Chem., 1987, (6), 521.

[9] Dewick, P.M. Medicinal Natural Products. John Wiley \& Sons: West Sussex, UK, 1997.

[10] Schulz, V.; Hansel, R.; Tyler, V. Rational Phytotherapy: a Physician's Guide to Herbal Medicine, $3^{\text {rd }}$ ed. Springer-Verlag: Berlin, 1998.

[11] Vidal Le Dictionnaire, 82e ed. version 1/3, -Issy-Les-Maulineaux, France, 2008, p. 905, p. 2119.

[12] Klenijnen, J.; Knipschild, P. Ginkgo biloba. Lancet, 1992, 340, 1136.

[13] Krieeglstein, J.; Beek, T.; Seibert, A. Influence of an extract of Ginkgo biloba on cerebral blood and metabolism. Life Sci., 1996, 39, 2327. 
[14] Hai, P.; Fen, L.Y.; Gang, S.S. Effects of Ginkgo biloba extract on acute cerebral ischemia in rats analyzed by magnetic resonance spectroscopy. Acta. Pharmacol. Sin., 2003, 24, 467.

[15] Blumenthal, M. Herb sales down 15 percent in mainstream market. HerbalGram., 2001, 51, 69.

[16] Sastre, J.; Millan, A.; Garcia de la Asuncion, J.; Pla, R.; Juan, G.; Pallardo; O'Connor, E.; Martin, J.A.; Droy-Lefaix, M.T.; Vina, J. A Ginkgo biloba extract (EGb 761) prevents mitochondrial aging by protecting against oxidative stress. Free Radic. Biol. Med., 1998, 24, 298.

[17] Kanowski, S.; Herrmann, W.M.; Stephan, K.; Wierich, W.; Horr, R. Proof of efficacy of the Ginkgo biloba special extract ( $G b \mathrm{E} 761$ ) in outpatients suffering from mild to moderate primary degenerative dementia of the Alzheimer type or multi-infarct dementia. Pharmacopsychiatry, 1996, 29, 47.

[18] Le Bars, P.L.; Katz, M.M.; Berman, N.; Itil, T.M.; Freedman, A.M.; Schatzberg, A.F. A placebo-controlled, double-blind, randomized trial of an extract of Ginkgo biloba for dementia. North American EGb Study Group. JAMA, 1997, 278, 1327.

[19] Hofferberth, B. The efficacy of EGb 761 in patients with senile dementia of the Alzheimer type, a double-blind, placebo-controlled study on different levels of investigation. Hum. Psychopharmacol., 1994, 9, 215

[20] Shan, S.J.; Su-Fen, Y.; Zheng-Qin, Y.; Qin, W.; An-Zheng, S.; Xie-Nan, H. Protective effect and mechanism of Ginkgo biloba leaf extracts for Parkinson disease induced by 1-methyl-4-phenyl1,2,3,6-tetrahydropyridine. Acta Pharmacol., 2001, 22, 1089.

[21] Louajri, A.; Harraga, S.; Godot, V.; Toubin, G. The effect of ginkgo biloba extract on free radical production in hypoxic rats. Biol. Pharm., 2001, 24, 710.

[22] Zhang, Y.Y.; Fen, L.P.; Duan, L.I. Effect of Ginkgo biloba leaf extract on electroencephalography of rat with cerebral ischemia and reperfusion. Acta. Pharmacol. Sin., 2003, 24, 157.

[23] Van Beek, T.A. Chemical analysis of Ginkgo biloba leaves and extracts. J. Chromatogr. A, 2002, 967, 21.

[24] Jensen, A.G.; Ndjoko, K.; Wolfender, J.L.; Hostettmann, K.; Camponovo, F.; Soldati, F. Liquid chromatography-atmospheric pressure chemical ionisation/mass spectrometry: a rapid and selective method for the quantitative determination of ginkgolides and bilobalide in ginkgo leaf extracts and phytopharmaceuticals. Phytochem. Anal., 2002, 13, 31.

[25] Li, W.; Fitzloff, J.F. Simultaneous determination of terpene lactones and flavonoid aglycones in Ginkgo biloba by highperformance liquid chromatography with evaporative light scattering detection. J. Pharm. Biomed. Anal., 2002, 30, 67.

[26] Lobstein-Guth, A.; Briancon-Scheid, F.; Anton, R. Analysis of terpenes from ginkgo biloba L. by high-performance liquid chromatography. J. Chromatogr., 1983, 267, 431.

[27] Mesbah, M.K.; Khalifa, S.I.; El-Gindy, A.; Tawfik, K.A. HPLC determination of certain flavonoids and terpene lactones in selected Ginkgo biloba L. phytopharmaceuticals. Il Farmaco, 2005, 60, 583.

[28] Teng B.P. Chemistry of ginkgolides. Braquet, P., Ed.; GinkgolidesChemistry, Biology, Pharmacology and Clinical Perspectives, J. R. Prous. Science: Barcelona, 1988, vol. 1, pp. 37-41.
[29] Van Beek, T.A.; Scheeren, H.A.; Rantio, T.; Melger, W.C.; Lelyveld, G.P. Determination of ginkgolides and bilobalide in Ginkgo biloba leaves, extracts and phytopharmaceuticals. $J$. Chromatogr., 1991, 543, 375.

[30] Lang, Q.; Yak, H.K.; Wai, C.M. Selective dissolution and one step separation of terpene trilactones in ginkgo leaf extracts for GC-FID determination. Talanta, 2001, 54, 673 .

[31] Lang, Q.; Wai, C.M. An extraction method for determination of ginkgolides and bilobalide in ginkgo leaf extracts. Anal. Chem. 1999, 71, 2929.

[32] USP 24, U.S. Pharmacopeial Convention, $25^{\text {th }}$ ed. Twinbrook Parkway, Rockville, MD, 1999, p. 2458

[33] Maillard, M.P.; Wolfender, J.L.; Hostettmann, K. Use of liquid chromatography- thermospray mass spectrometry in phytochemical analysis of crude plant extracts. J. Chromatogr., 1993, 647, 147.

[34] Deng, F.; Zito, S.W. Development and validation of a gas chromatographic-mass spectrometric method for simultaneous identification and quantification of marker compounds including bilobalide, ginkgolides and flavonoids in Ginkgo biloba L. extract and pharmaceutical preparations. J. Chromatogr. A, 2003, 986, 121.

[35] Tang, C.; Wei, X.; Yin, C. Analysis of ginkgolides and bilobalide in Ginkgo biloba L. extract injections by high-performance liquid chromatography with evaporative light scattering detection. $J$. Pharm. Biomed. Anal., 2003, 33, 811 .

[36] Dubber, M.J.; Kanfer, I. Determination of terpene trilactones in Ginkgo biloba solid oral dosage forms using HPLC with evaporative light scattering detection. J. Pharm. Biomed. Anal., 2006, $41,135$.

[37] Dubber, M.J.; Kanfer, I. Application of reverse-flow micellar electrokinetic chromatography for the simultaneous determination of flavonols and terpene trilactones in Ginkgo biloba dosage forms. J. Chromatogr. A, 2006, 1122, 266.

[38] Van Beek, T.A.; Van Veldhuizen, A.; Lelyveld, G.P.; Piron, I.; Lankhorst, P.P. Quantitation of bilobalide and ginkolides A, B, C and $\mathrm{J}$ by means of nuclear magnetic resonance spectroscopy. Phytochem. Anal., 1993, 4, 261.

[39] de Jager, L.S.; Perfetti, G.A.; Diachenko, G.W. Analysis of Ginkgolides, and Bilobalide in "Functional Food" Products using LC-APCI-MS. J. Pharm. Biomed. Anal., 2006, 41, 1552.

[40] Mauri, P.; Minoggio, M.; Iemoli, L.; Rossoni, G.; Morazzoni, P.; Bombardelli, E.; Pietta, P. Liquid chromatography/atmospheric pressure chemical ionization ion trap mass spectrometry of terpene lactones in plasma of animals. J. Pharm. Biomed. Anal., 2003, 32, 633.

[41] Balz, J.P.; Courtois, D.; Drieu, J.; Drieu, K., Reynoird, J.P.; Sohier, C.; Teng, B.P.; Touche' A.; Pe'tiard, V. Production of ginkgolides and bilobalide by Ginkgo biloba plants and tissue cultures. Planta Med., 1999, 65, 620 .

[42] Lolla, E.; Paletti, A.; Peterlongo, F. Gas chromatographic determination of ginkgolides and bilobalide in Ginkgo biloba leaves and extracts. Fitoterapia, 1998, 69, 513.

[43] Van Beek, T.A.; Taylir, L.T. Sample preparation of standardized extracts of Ginkgo biloba by supercritical fluid extraction. Phytochem. Anal., 1996, 7, 185. 